\title{
THE ROLE OF SOUTH AFRICAN TRADITIONAL HEALTH PRACTITIONERS IN THE TREATMENT OF HIV/AIDS: A STUDY OF THEIR PRACTICES AND USE OF HERBAL MEDICINES
}

\begin{abstract}
David Walwyn, BSc (Chem Eng), PhD
iThemba Pharmaceuticals, PO Box 21, Modderfontein, 1645, and Graduate School of Technology Management, University of Pretoria
\end{abstract} Boitumelo Maitshotlo, Nat Dip Food Service Management

iThemba Pharmaceuticals

Background. A large proportion of HIV-positive South Africans regularly consult traditional health practitioners (THPs) for their health care needs, despite evidence of negative interactions with antiretrovirals (ARVs) and no published peer-reviewed clinical evidence for the efficacy of traditional medicines in the treatment of HIV. We investigated the dominant practices of THPs towards HIV-positive patients and whether these practices have changed following widespread public awareness campaigns covering HIV and its treatment.

Method. The study used a semi-structured interviewer-administered questionnaire in the home language of the interviewee. A total of 52 THPs from four provinces (Gauteng, Limpopo, KwaZulu-Natal and Eastern Cape) were interviewed. Of the respondents 38\% were based in the rural areas, and 69\% classified themselves as inyangas, the remainder being sangomas.

Findings. All the THPs in the survey offered treatment for HIV, although only $20 \%$ claimed to be able to cure the disease; 88\% prepared their own medication, mostly from plant material, and sold their products as aqueous extracts in labelled bottles. None of these products had been systematically evaluated, and there was generally no record keeping, either of the patient or of the medicine itself. Quality control practices such as expiry dates, controlled storage conditions and batch records were totally unknown in our sample. Only 38\% of the THPs had received training on HIV/AIDS, although 75\% believed that they were well informed about the disease. Our own assessment was that only 50\% had a working knowledge of HIV; more disturbingly, 37\% believed that only traditional medicines should be used for its treatment and a further $50 \%$ believed that traditional medicines and ARVs can be taken simultaneously.

Interpretation. Despite ongoing public educational campaigns on HIV, some of which have specifically targeted THPs, the care of HIV-positive patients continues to be compromised by the traditional sector. Although some progress is evident, THP approaches to HIV treatment fail to conform to minimum standards proposed by the World Health Organization and other organisations, and represent a considerable challenge to the integration of THPs with the biomedical sector and the antiretroviral treatment programme in South Africa.

Arguments in favour of the incorporation of traditional health practitioners (THPs) into the overall health system are highly persuasive, ${ }^{1,2}$ especially in the context of South Africa's HIV epidemic and the inability of the public health sector to cover the health care needs of all HIV-positive patients. ${ }^{3}$ However, even those who support a more significant role for THPs have articulated a set of minimum standards that must be met as a pre-condition to this step, including:2,4,5

- the systematic evaluation of African traditional medicines (ATMs)

- the standardisation, processing and packaging of traditional medicines
- training on HIV to ensure a high level of prevention (of HIV) and care (of patients)

- the development of mechanisms to regulate the practice of traditional healing.

Over the past 3 years, Arvir Technologies, a publicfunded company that was established to validate and register a herbal medicine for the treatment of HIV based on ATMs, have been working on the first of these standards, namely the evaluation of ATMs, and more specifically on three plant extracts the use of which for the treatment of HIV/AIDS by THPs has been reported previously. ${ }^{6,7}$ This project has raised a number 
of important questions about the curative approaches and practices of THPs, and the supply channels for the plant-based ingredients of their medicines, as follows:

- Several studies ${ }^{8-11}$ have reported widespread consultation of THPs by people living with HIVI AIDS, reflecting a high level of trust in both traditional methods and ATMs; what is the basis of this support, and is there any evidence for the efficacy claims of THPs in their treatment of HIV (anecdotal or clinical)?

- The traditional health sector is known to be highly divergent; what are the dominant treatment approaches, and what are the trends in this regard?

- Regulation of the sector has recently been implemented through the promulgation of the Traditional Health Practitioners Act (No. 22 of 2007); to what extent has the Act changed the profile and conduct of the sector, and could this facilitate the introduction of a fully validated herbal medicine?

- Similarly, there have been several educational programmes to assist THPs to improve their understanding of the disease and their alignment with the goals of the antiretroviral (ARV) treatment (ART) programme in South Africa; have the programmes been successful in this regard?

- Allopathic and traditional medicines are typically seen as highly divergent; what is the level of interaction between the two sectors (biomedical and traditional health care), and are inter-sectoral referrals increasing?

In our research, we have attempted to answer these questions. The underlying intention was to develop an understanding of whether and how we could engage with the traditional health sector, and to define better approaches for improving the biomedical/traditional health interface.

\section{METHODS}

The study was undertaken using a semi-structured interview-administered questionnaire. It was conducted in four provinces of South Africa, and a total of 52 THPs were interviewed (Table I). The interviewees were identified by asking members of the community for information on the prominent THPs; once identified, the researcher explained the purpose of the study to the practitioner and requested permission for the interview.

\section{TABLE I. DISTRIBUTION OF INTERVIEWEES} BY PROVINCE

\begin{tabular}{|lc|}
\hline Province & No. of THPs participating \\
\hline Eastern Cape & 13 \\
Gauteng & 11 \\
KwaZulu-Natal & 15 \\
Limpopo & 13 \\
\hline
\end{tabular}

The questionnaire covered a range of areas including membership of a professional association (as prescribed by the Traditional Health Practitioners Act), the extent of training on HIV/AIDS already completed (including treatment with ARVs), the level of knowledge about the prevention, incidence, diagnosis and biomedical treatment of HIV/AIDS, the number of HIV-positive patients being treated, interaction with the biomedical sector (referrals and diagnosis), and the nature of the THP's treatment for HIV (including quality control of prescribed medicines).

\section{RESULTS}

\section{AN OVERVIEW OF THP PRACTICE AND HIV/AIDS}

The majority of THPs (69\%) in our study classified themselves as inyangas (herbalists); in other words, the source of their healing is considered to derive from their use of medicinal plants, as opposed to the approach of the sangomas (diviners), who rely more on divination for their healing approach. Diviners have different names in different regions of South Africa, depending on the dominant regional culture, including izangoma in Zulu, amagqira in Xhosa, ngaka in Northern Sotho and mungome in Venda, although the majority of South Africans refer collectively to this group of THPs as 'sangomas.'12,13

Except in the Eastern Cape and Gauteng (where the figure was only 50\%), most of the THPs in this study (79\%) were registered with a local THP association, although no practitioners were as yet registered in terms of the Traditional Health Practitioners Act (No. 22 of 2007). This and other observations lead to the inevitable conclusion that implementation of the Act since its promulgation has been almost non-existent in all regions of the country.

Not surprisingly, all the THPs in our study regularly see HIV-positive patients (between 3 and 10 patients per week). The THPs stated that most of their patients were women, but believed that males are the dominant transmitters of HIV/AIDS. In their view, the gender differences make it difficult to treat couples (either discordant or both HIV positive) because men do not talk openly about their HIV status and are therefore less likely to seek treatment.

\section{TRAINING AND KNOWLEDGE OF HIV/AIDS}

Only 38\% of the THPs had received HIV/AIDS training of one or more days; as a result, their knowledge of the disease was limited and influenced by a high level of suspicion of non-traditional interventions and biomedical strategies. For instance, some THPs still believe that there is no such thing as HIV/AIDS and continue to associate the symptoms of the disease with cultural notions of sickness, e.g. makgome in Pedi and isifusenhlu in Zulu, which require the performance of 
a cleansing ceremony for healing. In our assessment, which was based on answers to a standard set of questions about HIV, its treatment and its prevention, only $50 \%$ of respondents have a working knowledge of HIV, although 75\% feel that they were well informed about the disease and its symptoms (Fig. 1).

Although limited in the sample, THPs' ignorance and mistrust of condom use as a prevention strategy is of great concern. Some opinions expressed in this regard were:

'In the past there were no such things as condoms or HIV in our society; ever since condoms were brought to us there is a high rate of HIV/AIDS because they are infested with diseases. Proof is in that oil lubricant.'

'Condoms are contributing to the spread of HIV/ AIDS, for an example if you put condom in a sunny condition or pour hot water on it, you will notice worms coming out of that experiment. That is the very same worm they are referring to as AIDS.'

\section{DIAGNOSIS AND TREATMENT}

For the diagnosis of HIV, at least $69 \%$ of the THPs stated that they refer their patients to the biomedical sector for testing and require a positive HIV test result before the patient can start treatment (Fig. 2). The remaining practitioners use various approaches including THP observation, patient self-diagnosis and 'dreams and guidance from the ancestors'.

The modality of the treatment, even within the two separate groups of inyangas and sangomas, varied considerably. The latter often require patients to undergo a rigorous in-house cleansing and treatment routine. For instance, a THP in rural Eastern Cape, who sees HIV-positive clients from all over the country, has built a hospice in his yard for his patients. Inyangas, on the other hand, follow an approach of an initial consultation with fortnightly or monthly followup visits, depending on how quickly the prescribed treatments are completed.

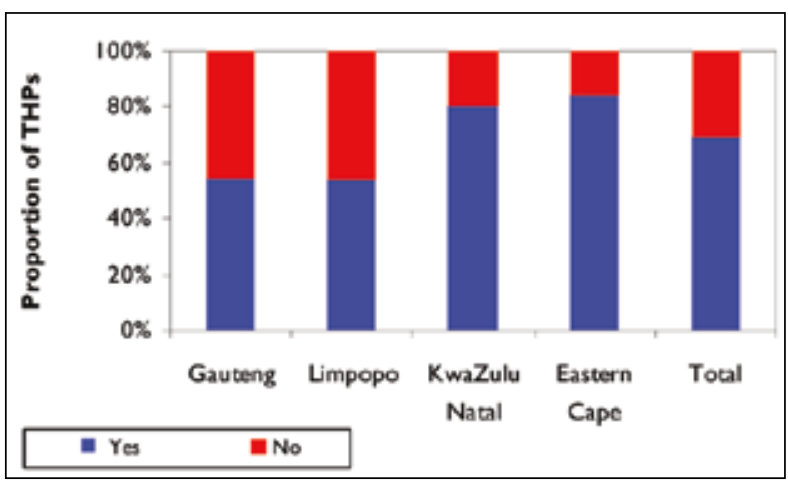

Fig. 2. THPs referring patients to clinics for HIV testing.

Of THPs in our sample $73 \%$ prescribe a herb-based solution called imbiza (Zulu) which is sold in a 2 litre or $750 \mathrm{ml}$ bottle and is claimed to cleanse or purify the blood. It was apparent from the research that a wide range of plants collected from different geographical regions and ecological systems are used in the preparation of the imbiza. It is claimed that the practitioners' understanding of plant medicinal properties is based on an extensive knowledge of their traditional use, obtained from the ancestors and passed down through oral tradition. Almost all the THPs (88\%) prepare their own medicine using the natural plant ingredients and a hot water infusion process.

The questionnaire specifically avoided detailed questions about the exact identity of the ingredients in order to protect any potential intellectual property. In some cases, however, this information was provided without any prompting and included a number of plants, the antiviral and immunomodulatory properties of which have already been reported., ${ }^{714-17}$ The plants were sourced from the local area (collected personally by the THP) and also from markets trading in medicinal plants (mostly in the major urban areas).

The recommended dosage varies according to a number of factors, as follows:

- stage of the disease (chronic stage $125 \mathrm{ml} 3$ times a day; bed-ridden patients $250 \mathrm{ml} 3$ times a day)

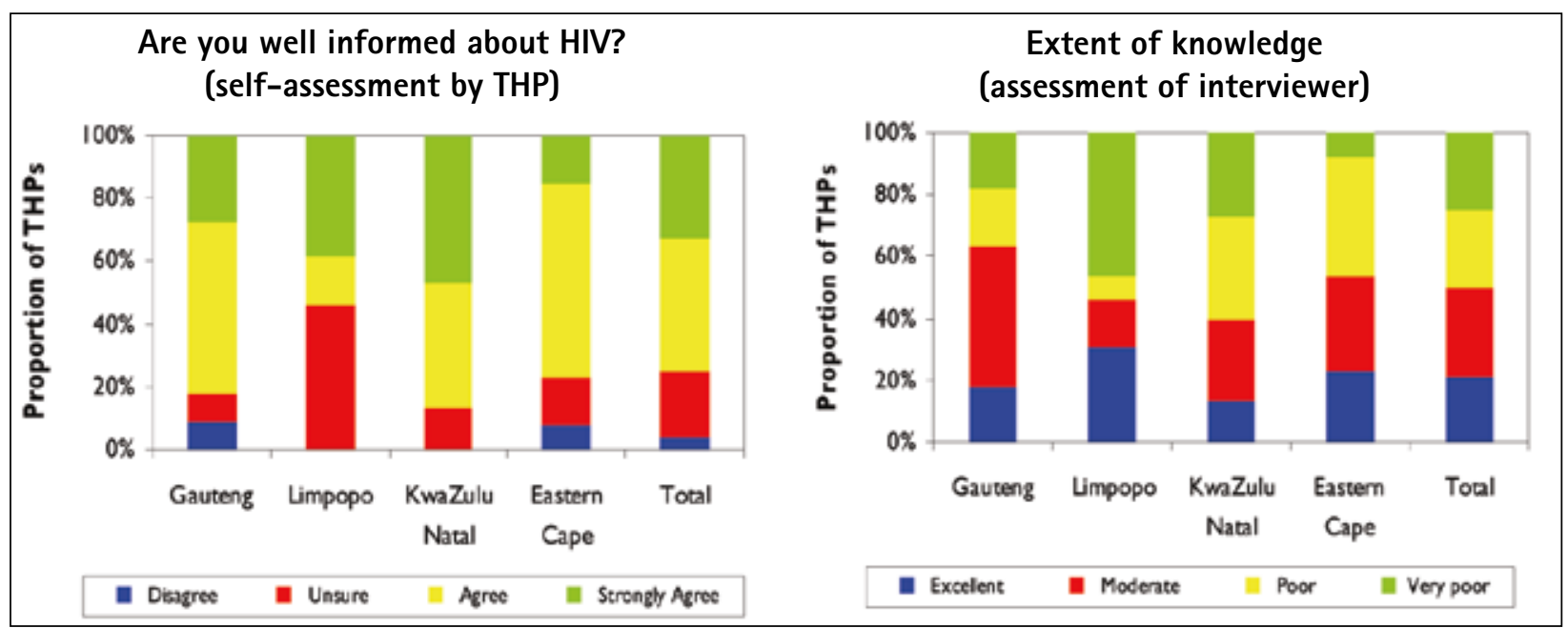

Fig. 1. Extent of knowledge (THPs and interviewer assessment). 
affordability to the patient (unemployed patients 3 times a day; employed patients 4 times a day)

- distance from which the patient comes (patients from far places are given a 5 litre bottle and take $60 \mathrm{ml}$ of the medicine 3 times a day).

The cost of medicine also varies from a minimum of R200 to a maximum of R2 800 (Fig. 3). The treatment cost is determined by the following:

- the local reputation of the THP and hence patient belief in the efficacy of ATMs (the better the reputation, the more expensive the treatment)

- the variety of herbs used (the more herbs, the higher the price)

- the location of the practice (prices in the rural areas are generally lower).

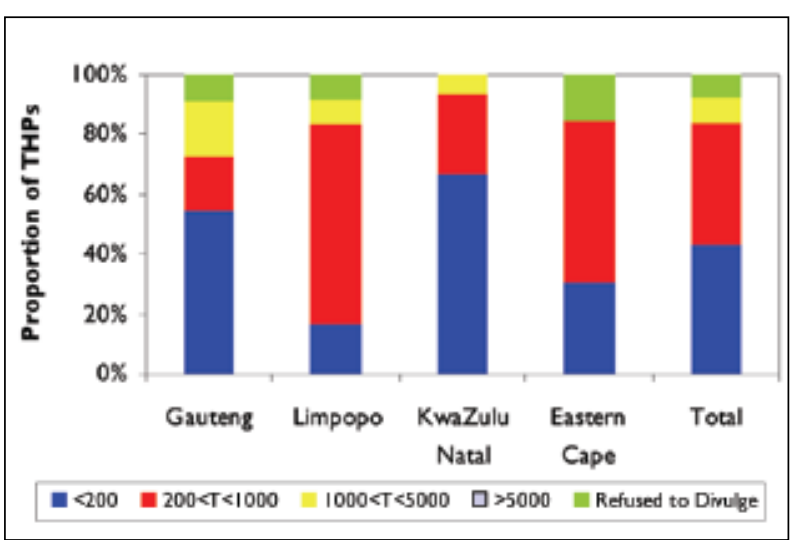

Fig. 3. Cost of traditional medicines for the treatment of HIV.

All the THPs in our survey were confident about the efficacy of their treatment for both HIV (20\% claim to be able to cure HIV; the remainder to treat the infection) and HIV/AIDS-related opportunistic infections, although we were shown no recorded evidence for these claims. When asked to show evidence to support their statement, a number of claims were made including verbal reports of increases in CD4 count and patient well-being. As noted later, only 17\% of THPs keep any patient records and track HIV status.

Based on our own research and the work of other drug discovery organisations, the basis for claims relating to most of the plants is highly questionable. This statement is supported by two main arguments derived from an extensive evaluation of the antiretroviral properties of plant extracts, as follows: ${ }^{6}$

- Most of the plant-based traditional medicines are prepared through hot water infusion of dried plant material (leaves, roots, bark, etc.); the resultant solutions contain at most $0.5 \%$ of extracted solids, so that a $250 \mathrm{ml}$ dose in turn contains the equivalent of $1.25 \mathrm{~g}$ solid material. The concentration of the active ingredient(s) varies considerably depending on the plant itself and in many cases these ingredients have not been properly identified. However, our work with three local products indicates that the active ingredient(s) are at most 5\% by mass of the total extract. In other words, a patient taking a $250 \mathrm{ml}$ dose of a herbal infusion (the highest dose recorded in our study) will be consuming not more than $63 \mathrm{mg}$ of active ingredient(s) per dose, or 188 mg per day.

- A wide range of specific antiretroviral activities for plant-based natural products (and other compounds) has been reported in the literature, ${ }^{14-17}$ but even the most active natural compounds have activities no better than between 1 and $5 \mu \mathrm{M}$ based on a range of in vitro cell-based assays. These values are between 100 and 1000 times less active than the equivalent allopathic medicines such as zidovudine, efavirenz and tenofovir (Tables II and III). In other words, a patient taking a traditional medicine for the treatment of HIV will be receiving on average less than $0.5 \%$ of a therapeutic dose. This calculation does make some assumptions about the bio-availability of the natural product(s) but is a reasonable estimate of effective dose and its comparison with the registered antiretroviral products.

In summary, although many plant extracts have a measurable level of antiretroviral activity (as determined in cell-based assays), this activity is many times lower than that of the synthetic products. Considering that HIV patients on traditional medicines are taking on average less than $150 \mathrm{mg}$ of the active compound(s), the net dose is only a fraction of the required therapeutic dose, and the possibility that a level of viral control is achieved is remote.

\section{QUALITY CONTROL, EFFICACY AND PATIENT RECORDS}

Most of the THPs (69\%) claimed that their medication is of good quality and that there is no need for batch records, expiry dates or quality assessment of the ingredients/final product, which are standard concepts in the preparation of allopathic medicines. When asked to explain the reason for the difference, the THPs noted that 'unlike Western medicine our medicine doesn't have any preservatives; therefore there is no need to include expiry dates because natural plants don't expire'. In addition, very few THPs label their products.

In terms of patient records, as has been stated previously, only $17 \%$ of THPs keep any form of records, with the actual proportion varying from 26\% in Gauteng to $8 \%$ in the Eastern Cape. As a result, no conclusive results can be derived from analysis of the treatment outcomes since these cannot be supported by documented evidence including health assessments, viral loads and other indicators of disease progression in HIV-positive patients. It is clear that this is one important area in which training can play a major role in upgrading the quality of care being provided by THPs in South Africa. 
TABLE II. ANTIRETROVIRAL ACTIVITY AND ACTUAL DOSAGE OF SEVERAL SYNTHETIC PRODUCTS*

\begin{tabular}{|llllll|}
\hline Drug name & Class & $\mathbf{I C}_{50}(\mu \mathrm{M})$ & $\mathrm{TC}_{50}(\mu \mathrm{M})$ & Therapeutic index & Dosage $(\mathbf{m g} / \mathbf{d})$ \\
\hline Efavirenz & $\mathrm{NNRTI}$ & 0.0015 & 80 & 53333 & 600 \\
Maraviroc & $\mathrm{EI}$ & 0.001 & 25 & 20161 & 600 \\
Lamivudine & $\mathrm{NRTI}$ & 0.07 & 360 & 5143 & 300 \\
Nevirapine & $\mathrm{NNRTI}$ & 0.029 & 320 & 11034 & 400 \\
Stavudine & $\mathrm{NRTI}$ & 0.03 & 100 & 3333 & 60 \\
Zidovudine & $\mathrm{NRTI}$ & 0.002 & 32 & 16000 & 600 \\
Emtricitabine & $\mathrm{NRTI}$ & 0.04 & 150 & 3750 & 200 \\
Tenofovir & $\mathrm{NRTI}$ & 0.005 & 29 & 5800 & 1600 \\
Indinavir & $\mathrm{PI}$ & 0.014 & 32 & 2286 & 1600 \\
*Data for all the synthetic and natural products have been obtained directly from the National Institute of Health's database, & which lists the anti-HIV activity of \\
over 170 000 compounds based on information in the literature. Where possible the values for the PBMC assay have been used. & \\
NNRTI = non-nucleoside reverse transcriptase inhibitor; El = entry inhibitor; NRTI = nucleoside reverse transcriptase inhibitor; PI = protease inhibitor.
\end{tabular}

TABLE III. ANTIRETROVIRAL ACTIVITY AND ESTIMATED DOSAGE OF SEVERAL NATURAL PRODUCTS*

\begin{tabular}{|c|c|c|c|c|c|}
\hline Plant & Active ingredient & $\mathrm{IC}_{50}(\mu \mathrm{M})$ & $\mathrm{TC}_{50}(\mu \mathrm{M})$ & Therapeutic index & $\begin{array}{l}\text { Estimated } \\
\text { dosage }(\mathrm{mg} / \mathrm{d})\end{array}$ \\
\hline \multirow{3}{*}{$\begin{array}{l}\text { Syzygium claviflorum } \\
\text { Lobostemon trigonus }^{6}\end{array}$} & Betulinic acid & 1.4 & 13 & 9 & 60000 \\
\hline & Unknown (data shown & & & & \\
\hline & for crude extract) & $\sim 2.5$ & 2000 & 800 & 150000 \\
\hline Curcuma longa & Curcumin & 5.0 & 10.0 & 2 & 100000 \\
\hline Inula britannica & 3,5-di-0-caffeoylquinic acid & 2.0 & 486 & 243 & 40000 \\
\hline Calophyllum lanigerum & Calanolide A & 0.1 & 10 & 100 & 8000 \\
\hline
\end{tabular}

\section{INTERACTION WITH THE BIOMEDICAL HEALTH CARE SECTOR}

When asked about the possibility of interaction with other health practitioners, THPs believed that nurses undermine their work and do not accept the efficacy of their treatment. As a result, THPs are reluctant to refer their patients to the biomedical sector for ART. A similar observation has been reported in other studies. ${ }^{19}$

The THPs indicated that unlike nurses and doctors they do not take the patients off ART but encourage their patients to take both ATMs and ARVs, since they believe that ARVs are also made from muti (Fig. 4). At least $50 \%$ of the THPs participating in our study showed little interest in learning or working with the biomedical sector and several openly criticised the allopathic approach, making statements such as 'doctors kill patients with their ARVs'.

\section{DISCUSSION}

The results of this study have shown that many THPs offer treatment for HIV/AIDS despite a somewhat limited understanding of the virus, the symptoms of infection and its treatment. Furthermore, the medicines are expensive relative to both patient incomes and the biomedical equivalents, have no recorded evidence of efficacy, and are not quality controlled in most respects.

Despite these factors, many patients continue to consult THPs for HIV-related illness and to use traditional medicines. The persistence of this support is due to many factors, including:

- an ongoing belief in the efficacy of traditional medicines, which is considered to have been passed down through generations from the ancestors

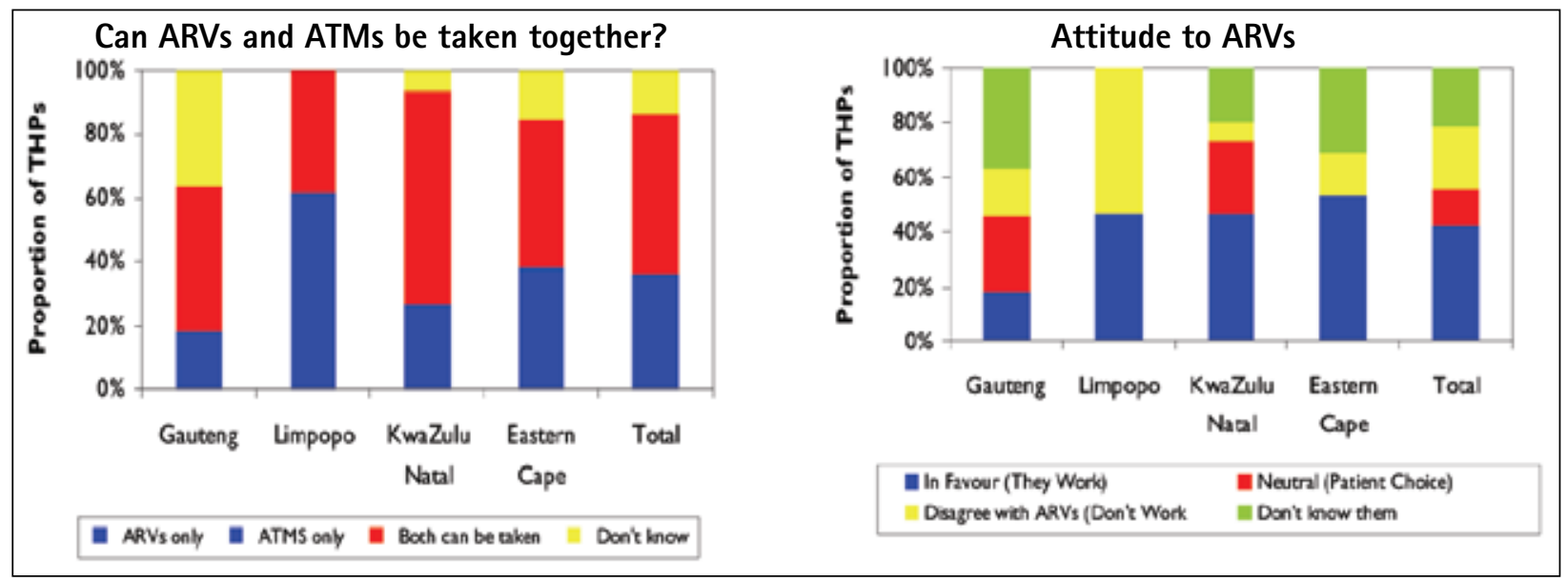

Fig. 4. THPs' attitudes to ARV treatment. 
- considerable competition between the two treatment methodologies, especially by the THPs, who openly discredit the conventional sector in various ways (referral of a patient to the tertiary sector by a THP is in many respects a direct conflict of interest)

- the confidentiality of consulting a traditional healer (there is a belief that information such as the patient's HIV status is not secure in the biomedical sector)

- the perceived uncaring, unsympathetic and impersonal approach of the biomedical sector to patients, which is humiliating and hurtful to the patient; doctors are considered to give inadequate time to patient consultation

- the side-effects of ARVs and the fact that these medications are not a cure, but have to be taken forever

- lack of regulation in the sector, which encourages illegal and opportunistic practices including wild claims about treatment efficacy; in some cases the more bizarre the treatment, the greater the number of patients.

These problems are well known in the sector, and other authors have identified a set of minimum standards that require urgent implementation. ${ }^{9}$ Our study has shown that little progress has been made with implementation of these standards. For instance:

- Although all of the THPs claim to treat HIV (and $20 \%$ to cure the disease), none of the treatments have been systematically evaluated.

- The majority (88\%) of the THPs interviewed prepare their own medication but fail to keep patient records or batch data for each preparation. Most of the products are sold in labelled bottles as liquids, and none have any expiry dates on the labels. The opinion of the THPs is that plants do not expire; there is therefore no need for proper packaging or controlled storage conditions.

- Of the THPs in our sample, 38\% have received training on HIV/AIDS and 75\% believe that they are well informed about the disease; however, based on their replies to a few rudimentary questions about the disease, our assessment was that only $50 \%$ have a working knowledge of HIV.

- Perhaps more disturbingly, 37\% believe that only ATMs should be used for the treatment of HIV and a further 50\% believe that both ATMs and ARVs can be taken simultaneously.

It is apparent that many THPs play a role in HIV prevention and care by referring patients for HIV testing (69\% of THPs), counselling patients with HIV and opportunistic infections, and distributing condoms to their patients (62\% of the total). Our study makes it clear that this role is sub-optimal and could be improved through further training and regulation.
Despite the fact that such regulation has recently been implemented by promulgation of the Traditional Health Practitioners Act, and that $79 \%$ of our sample of THPs were registered with a THP association, the Interim Traditional Health Practitioners Council of South Africa is not yet operational and there is no quality control in the sector or enforcement of the legislation.

With respect to capacity development of THPs, it is recommended that training in the following areas should be urgently addressed:

- an understanding of the HIV and the pathology of the disease

- the adoption of safe sexual practices and use of biomedical prevention methods

- quality control of traditional medicines, including concepts such as expiry and variability of raw materials

- the dangers of taking ATMs and ARVs together

- identification of symptoms of HIV infection to assist with counselling and treatment, including referral to the biomedical sector.

\section{CONCLUSION}

In conclusion, it will be a major challenge to use the THP network for distribution of a registered herbal medicine owing to ongoing ignorance regarding HIV, a high rate of illiteracy among THPs especially in rural areas, and local cultural beliefs that may prevent adoption of biomedical approaches. Nevertheless, the extensive network of THPs and ongoing confidence in their abilities within local communities suggest that efforts to address these challenges will be worthwhile.

Authors' contributions. This article was written by Dr David Walwyn. The field work was undertaken by Ms Biotumelo Maitshotlo, who also contributed to the preparation of the manuscript with written material in certain sections, graphics and checking of the contents.

Conflict of interest. There is no conflict of interest for either author. The work was undertaken to understand the dynamics of the traditional health care sector in South Africa with respect to the treatment of HIV/AIDS, and to consider the suitability of this sector for the distribution of a herbal medicine. These objectives are clearly stated in the text of the article.

Acknowledgements. Payment of the salary of Ms Maitshotlo and overall funding of Arvir Technologies by LIFElab are gratefully acknowledged. 


\section{REFERENCES}

1. Wreford, J. Missing each other: problems and potential for collaborative efforts between biomedical and traditional healers in the time of AIDS. Social Dynamics 2005; 31(2): 55-89.

2. World Health Assembly. Resolution of the 62nd World Health Assembly on Traditional Medicine. Document Number EB124.R9. Geneva: World Health Assembly, 2009.

3. Cleary S. Scaling-up access to ART: confronting affordability and sustainability. Presented at the 4th South African AIDS Conference, Durban, 31 March - 3 April 2009.

4. Dickinson D. Traditional healers and company HIV/AIDS programmes. African Journal of AIDS Research 2008; 7(3): 281-291.

5. Southern African Development Community. Article 20 of the SADC Protocol on Health. Adopted in August 1999, Maputo. http://www.doh.gov.za/department/ sadc/docs/protocol99.html (accessed 25 June 2009).

6. Walwyn D. Herbal medicines for the treatment of HIV: Fanciful notion or real possibility? Poster presented at the 4th South African AIDS Conference, Durban, 31 March - 3 April 2009.

7. Bessong PO, Obi CL. Ethnopharmacology of human immunodeficiency virus in South Africa - a minireview. African Journal of Biotechnology 2006; 5(19): 16931699.

8. Peltzer K, Friend-du Preez N, Ramlagan S, Fomundam H. Use of traditional complementary and alternative medicine for HIV patients in KwaZulu-Natal, South Africa. BMC Public Health 2008; 8: 255-269.

9. Homsy J, King R, Tenywa J, Kyeyune $P$, Opio A, Balaba D. Defining the minimum standards of practice for incorporating African traditional medicine into HIV/AIDS prevention, care and support: a regional initiative in Eastern and Southern Africa. African Journal of Traditional, Complementary and Alternative Medicines 2004; 10(5): 905-910.
10. Mngqundaniso N, Peltzer K. Patients consulting traditional health practitioners in the context of HIV/AIDS in urban areas in KwaZulu Natal, South Africa. African Journal of Traditional, Complementary and Alternative Medicine 2008; 5(4): 370 379 .

11. Babb DA, Pemba L, Seatlanyane P, Charalambous S, Churchyard GJ, Grant AD. Use of traditional medicine by HIV-infected individuals in South Africa in the era of antiretroviral therapy. Psychol Health Med 2007; 12(3): 314-320.

12. Wreford J. Sincedisa - We can help: a literature review of current practice involving traditional African healers in biomedical HIV/AIDS interventions in South Africa. Social Dynamics 2005; 31(2): 90-117.

13. Pretorius E. Traditional healers. In: South African Health Review. 5th ed. Durban: Health Systems Trust, 1999: 249-256.

14. Singh IP, Bharate SB, Bhutani KK. Anti-HIV natural products. Current Science 2005 89(2): 269-290.

15. De Clercq E. Current lead natural products for the chemotherapy of human immunodeficiency virus (HIV) infection. Med Res Rev 2000; 20: 323-349.

16. Cos $P$, Maes $L$, Van den Berghe $D$, Hermans N, Pieters L, Vlietinck A. Plant substances as anti-HIV agents selected according to their putative mechanism of action. J Nat Prod 2004; 67: 284-293.

17. Yu D, Morris-Natschke SL, Lee K-H. New developments in natural products-based anti-AIDS research. Med Res Rev 2007; 27(1): 108-132.

18. National Institute of Health. http://chemdb.niaid.nih.gov/struct_search/ivt/ivt_ search.asp (accessed 26 June 2009).

19. Mngqundaniso N, Peltzer K. Traditional healers and nurses: a qualitative study on their role on sexual transmitted infections including HIV and AIDS in KwaZulu Natal, South Africa. African Journal of Traditional, Complementary and Alternative Medicine 2008; 5(4): 380-386. 